\title{
Model-Switched Beamformer with Large Dynamic Range
}

\author{
Zeng Hao, Xing Qian, Yang Qingkun, and Feng Linfeng
}

Chongqing University, Chongqing 400044, China

Correspondence should be addressed to Zeng Hao; haoz@cqu.edu.cn

Received 25 March 2014; Revised 20 June 2014; Accepted 20 August 2014; Published 13 October 2014

Academic Editor: Lorenzo Crocco

Copyright (C) 2014 Zeng Hao et al. This is an open access article distributed under the Creative Commons Attribution License, which permits unrestricted use, distribution, and reproduction in any medium, provided the original work is properly cited.

\begin{abstract}
The strong desired signal will be mitigated due to "self-nulling" for the adaptive beamformer, even if the array calibration is used. The proposed methodology switches the models between phased array and adaptive array. In general, the system utilizes Frost adaptive beamforming. However, it will be switched to phased array if the "self-nulling" appears. According to the estimation of the array pattern at the direction of desired signal, we can determine if the "self-nulling" happens. The new approach is much easier to implement compared with the various robust beamforming algorithms.
\end{abstract}

\section{Introduction}

Adaptive beamformer, also named as adaptive array and adaptive spatial filter, plays important function in the cochannel interference mitigation for wireless communication, radar, sonar, and so forth. Compared with the blind beamforming, the nonblind beamforming attracts more interest, because its complexity is much lower. Frost algorithm in [1] is used most widely for minimum variance distortionless response (MVDR) beamformer, where the direction of arrival (DOA) of the desired signal is required to be known in order to calculate the weight vector. However, various array errors will result in the fact that the estimated DOA of desired signal vibrates from the actual one. When the power is large, the desired signal will be rejected deeply as interference. This phenomenon is called "self-nulling." Obviously, "self-nulling" makes the allowable dynamic range decrease for the desired signal.

Array errors stem from many facets. Documents $[2,3]$ analyze the performance of MVDR in presence of steering vector errors. Mutual coupling error is investigated for beamformer in $[4,5]$. Channel response errors are illustrated in $[6,7]$. Generally, array errors are overcome through two methods, namely, array calibration and robust adaptive beamforming. The literatures $[8,9]$ introduce some calibration methodologies to deal with the deterministic error, such as amplitude and phase errors in RF front-end. Furthermore, numerous robust beamformers are studied to combat the stochastic error. The strategies in $[10,11]$ are based on diagonal load where the white noise is added into the received signal on purpose. However, it is hard to determine the load value. Eigen structure [12] is applied in robust beamforming. Linear and nonlinear constraint algorithms in $[13,14]$ could obtain high robustness. Nevertheless, all these algorithms are too complicated to implement in real time through FPGA and DSPs devices.

We propose a model-switched beamformer, which works in two models, namely, phased array and adaptive array. The new methodology calculates the derivative of array pattern obtained by Frost algorithm. If the derivative at the estimated DOA of desired signal is larger than a determined threshold value, the "self-nulling" happens and beamformer chooses the phased array model. Otherwise, the weight vector of Frost algorithm is employed. Although the interferences cannot be rejected totally by phased array, generally, the beamformer could get high signal-to-interference-plus-noise ratio (SINR) response due to the strong desired signal. Even if the SINR is low, other technologies such as spread spectrum [15] can be used to deal with the interferences from the practice point of view.

This paper is organized as follows. Section 2 describes the system model. Section 3 presents the proposed methodology in detail. In Section 4, simulations and results are illustrated. And the conclusion is in Section 5. 


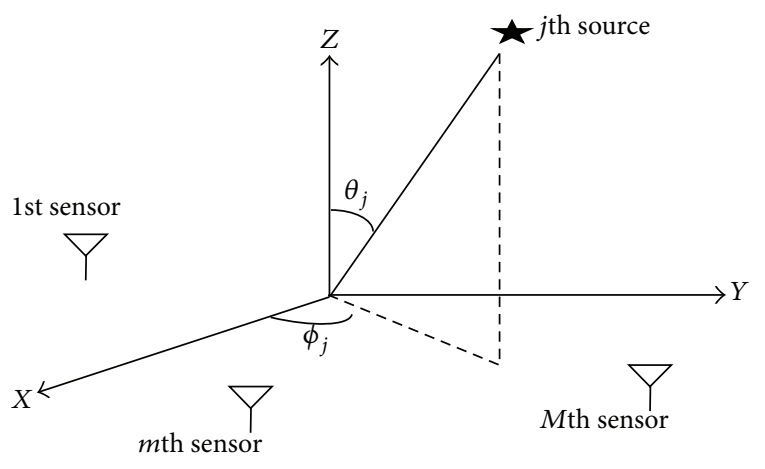

FIgURE 1: Sensors and sources positions.

\section{System Model}

2.1. Signal Model. As illustrated in Figure 1, arbitrary planar array with $M$ sensors lies in $X-Y$ planes. All sensors have the same characteristics and are isotropic. The coordinate of the $m$ th sensor is denoted by three-element vector $\mathbf{p}_{m}$, $m=1, \ldots, M$. Generally, the uniform arrays are used widely in practice such as uniform circular array and planar array. There are $J+1$ narrowband far field waves $s_{j}(t)$ with power $\sigma_{j}^{2}$, impinging from the elevation angle $\theta_{j}$ and azimuth angle $\phi_{j}$, respectively, $j=0,1, \ldots, J$. We assume that $s_{0}(t)$ is the desired signal and the others are interferences, where $J+1<M$. For convenience, signal at origin of coordination is given by

$$
s_{j}(t)=m_{j}(t) e^{j \omega t},
$$

where the $m_{j}(t)$ is the complex envelop and $\omega$ is the angular frequency. The received signal vector of the array is given by

$$
\mathbf{x}(t)=\sum_{j=0}^{J} s_{j}(t) \mathbf{v}_{j}+\mathbf{n}(t)
$$

$\mathbf{n}(t)$ is the i.i.d. Gaussian random noise vector with zero mean and covariance $\sigma_{n}^{2}$. And $\mathbf{v}_{j}$ is the direction vector of the $j$ th signal, which can be expressed as

$$
\mathbf{v}_{j}\left(\theta_{j}, \phi_{j}\right)=\left[\begin{array}{lll}
e^{-j \omega \tau_{j 1}} & \cdots & e^{-j \omega \tau_{j M}}
\end{array}\right]^{T},
$$

where $\tau_{j m}$ is the propagation delay of $s_{j}(t)$ between the $m$ th sensor and origin of coordination. " $T$ " is the transpose operator.

2.2. Phased Array. The framework of beamforming is shown in Figure 2, where we do not consider how to generate weight vector. The output of the beamformer is

$$
y(t)=\mathbf{w}^{H} \mathbf{x}(t)
$$

Operator " $H$ " represents Hermitian transpose. For phased array, the weight vector is decided by the DOA of desired signal; that is,

$$
\mathbf{w}_{P}=\mathbf{v}_{0}\left(\theta_{0}, \phi_{0}\right)
$$

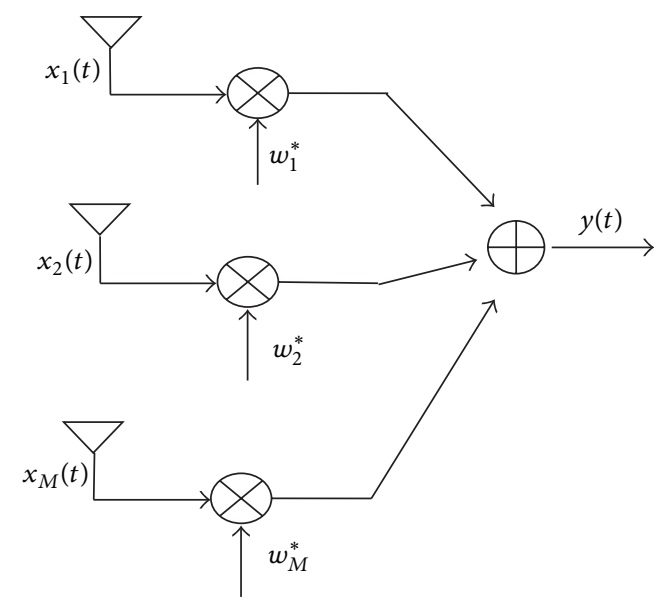

FiguRE 2: Beamforming structure.

According to (3), each element of the weight vector only adjusts the phase of received signals. And the main lobe of the array pattern is steered to direction $\left(\theta_{0}, \phi_{0}\right)$. The interferences will be degraded only by the side lobes. According to the array antenna theory, the array pattern is given by

$$
B(\theta, \phi)=\left|\mathbf{w}^{H} \mathbf{v}(\theta, \phi)\right| \text {. }
$$

2.3. Adaptive Array. Different from the phased array, the weight vector of adaptive array adjusts both amplitude and phase of the received signals. Then the main lobe is pointed to the desired signal and nulls are steered to all interferences simultaneously. The optimal weight vector for MVDR [16] is given by

$$
\mathbf{w}_{A}=\frac{\mathbf{R}^{-1} \mathbf{v}_{0}\left(\theta_{0}, \phi_{0}\right)}{\mathbf{v}_{0}^{H}\left(\theta_{0}, \phi_{0}\right) \mathbf{R}^{-1} \mathbf{v}_{0}\left(\theta_{0}, \phi_{0}\right)},
$$

where $\mathbf{R}$ denotes the signal covariance matrix. Because the signal is orthogonal to noise, we get

$$
\mathbf{R}=\sum_{j=0}^{J} \sigma_{j}^{2} \mathbf{v}_{j}^{H}\left(\theta_{j}, \phi_{j}\right) \mathbf{v}_{j}\left(\theta_{j}, \phi_{j}\right)+\sigma_{n}^{2} \mathbf{I} .
$$

Frost algorithm computes the weight vector through iteration. The iterative equation in [16] is

$$
\mathbf{w}(k+1)=\mathbf{P}_{c}\left[\mathbf{w}(k)-\alpha \mathbf{x}(k+1) y^{*}(k)\right]+\frac{\mathbf{v}_{0}\left(\theta_{0}, \phi_{0}\right)}{M},
$$

where

$$
\begin{gathered}
\mathbf{P}_{c}=\mathbf{I}-\mathbf{v}_{0}\left(\mathbf{v}_{0}^{H} \mathbf{v}_{0}\right)^{-1} \mathbf{v}_{0}^{H} \\
y(k)=\mathbf{w}^{H}(k) \mathbf{x}(k) .
\end{gathered}
$$

And $\alpha$ is the step size, I represents unit matrix, and $\mathbf{x}(k)$ denotes the $k$ th signal snapshot. The output of beamformer is also described as (4) and it obtains the maximum SINR after the weight vector converges. 


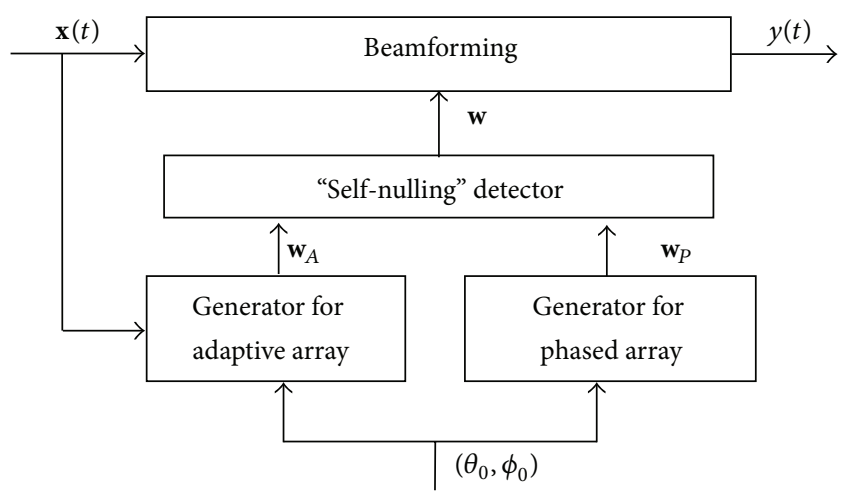

FIGURE 3: Model-switched beamformer.

\section{Proposed Model-Switched Beamformer}

For idealistic Frost MVDR beamformer in $[1,16]$, the maximum in array pattern is at $\left(\theta_{0}, \phi_{0}\right)$ and the value is $0 \mathrm{~dB}$ since the weight vector should satisfy the constraint of

$$
\mathbf{w}^{H} \mathbf{v}_{0}\left(\theta_{0}, \phi_{0}\right)=1 \text {. }
$$

Obviously, it is antenna gain at the centre of the main lobe. However, the actual DOA $\left(\bar{\theta}_{0}, \bar{\phi}_{0}\right)$ of the desired signal is different from the estimated DOA $\left(\widehat{\theta}_{0}, \widehat{\phi}_{0}\right)$ used in (9). When "self-nulling" happens, the desired signal is viewed as interference and the null appears at $\left(\bar{\theta}_{0}, \bar{\phi}_{0}\right)$ in array pattern, although constraint (11) is kept. Furthermore, the stronger the signal is, the deeper the null is. But it should be pointed out that "self-nulling" will not happen until the power of the desired signal reaches a certain value. And this value is related to array errors, signal parameters, and structure of array as well.

A model-switched beamformer is proposed in order to keep the high SINR output. As illustrated by Figure 3, the system consists of four parts: beamforming module, Frost weight vector generator, phased array weight vector generator, and "self-nulling" detector. We assume that the received signal $\mathbf{x}(t)$ and $\left(\widehat{\theta}_{0}, \widehat{\phi}_{0}\right)$ are known in the system.

In Figure 3, the weight vector generator for adaptive array utilizes the Frost algorithm and transfers the converged weight vector $\mathbf{w}_{A}$ to "self-nulling" detector. Then the detector judges whether "self-nulling" appears. As discussed above, the array pattern of beamformer will be smooth at $\left(\widehat{\theta}_{0}, \widehat{\phi}_{0}\right)$ without "self-nulling." This phenomenon will be illustrated in Section 4 . So the partial derivative $D\left(\widehat{\theta}_{0}, \widehat{\phi}_{0}\right)$ of array pattern at $\left(\widehat{\theta}_{0}, \widehat{\phi}_{0}\right)$ could be used to detect "self-nulling." Setting a small positive threshold $\varepsilon$ and if the following condition is satisfied,

$$
\begin{aligned}
D\left(\widehat{\theta}_{0}, \widehat{\phi}_{0}\right) & =\left.\left|\frac{\partial^{2} B(\theta, \phi)}{\partial \theta \cdot \partial \phi}\right|\right|_{\theta=\widehat{\theta}_{0}, \phi=\widehat{\phi}_{0}} \\
& =\left.\left|\frac{\partial^{2}\left|\mathbf{w}^{H} \mathbf{v}_{0}(\theta, \phi)\right|}{\partial \theta \cdot \partial \phi}\right|\right|_{\theta=\widehat{\theta}_{0}, \phi=\widehat{\phi}_{0}}>\varepsilon,
\end{aligned}
$$

it can be determined that "self-nulling" appears. Then the detector in Figure 3 chooses the phased array weight vector $\mathbf{w}_{P}$ for beamforming; otherwise, $\mathbf{w}_{A}$ is chosen. The optimal $\varepsilon$ is decided by the depth of null and DOA error. According to the experiments, $\varepsilon$ is from 1 to 5 for array with sensors less than ten.

The $D\left(\widehat{\theta}_{0}, \widehat{\phi}_{0}\right)$ can be estimated through the weight vector snapshot $\mathbf{w}(k)$. For uniform linear array, we set angle increment $\delta$ and

$$
0<\delta<\frac{\left|\bar{\theta}_{0}-\widehat{\theta}_{0}\right|}{2} .
$$

Then the estimation of (12) becomes

$$
\widehat{D}(k)=\left|\frac{\left|\mathbf{w}^{H}(k) \mathbf{v}_{0}\left(\widehat{\theta}_{0}+\delta\right)\right|-\left|\mathbf{w}^{H}(k) \mathbf{v}_{0}\left(\widehat{\theta}_{0}-\delta\right)\right|}{2 \delta}\right|>\varepsilon .
$$

We can take the average derivative to increase the accuracy by $K$ adjacent weight vector snapshots; that is,

$$
\begin{aligned}
\frac{1}{K} \sum_{k=k_{0}}^{k_{0}+K} \widehat{D}(k)=\frac{1}{2 K \delta} \sum_{k=k_{0}}^{k_{0}+K} & || \mathbf{w}^{H}(k) \mathbf{v}_{0}\left(\widehat{\theta}_{0}+\delta\right) \mid \\
& -\mid \mathbf{w}^{H}(k) \mathbf{v}_{0}\left(\widehat{\theta}_{0}-\delta\right) \|>\varepsilon
\end{aligned}
$$

In addition, the situation of planar array is more complicated than linear array. The derivative is estimated as

$$
\begin{aligned}
\widehat{D}_{\theta}(k) & =\left|\frac{\left|\mathbf{w}^{H}(k) \mathbf{v}_{0}\left(\widehat{\theta}_{0}+\delta, \widehat{\phi}_{0}\right)\right|-\left|\mathbf{w}^{H}(k) \mathbf{v}_{0}\left(\widehat{\theta}_{0}-\delta, \widehat{\phi}_{0}\right)\right|}{2 \delta}\right| \\
& >\varepsilon \\
\widehat{D}_{\phi}(k) & =\left|\frac{\left|\mathbf{w}^{H}(k) \mathbf{v}_{0}\left(\widehat{\theta}_{0}, \widehat{\phi}_{0}+\delta\right)\right|-\left|\mathbf{w}^{H}(k) \mathbf{v}_{0}\left(\widehat{\theta}_{0}, \widehat{\phi}_{0}-\delta\right)\right|}{2 \delta}\right| \\
& >\varepsilon .
\end{aligned}
$$

If either of the above two conditions is satisfied, the "selfnulling" exists. Of course, the average estimation also can be utilized. So (16) are rewritten as

$$
\begin{gathered}
\frac{1}{K} \sum_{k=k_{0}}^{k_{0}+K} \widehat{D}_{\theta}(k)>\varepsilon \\
\frac{1}{K} \sum_{k=k_{0}}^{k_{0}+K} \widehat{D}_{\phi}(k)>\varepsilon .
\end{gathered}
$$

\section{Simulations and Results}

We explore the simulation on the scenarios that there are one desired signal and one interference impinging on a uniform 8 -element linear array. The element spacing is half wavelength 


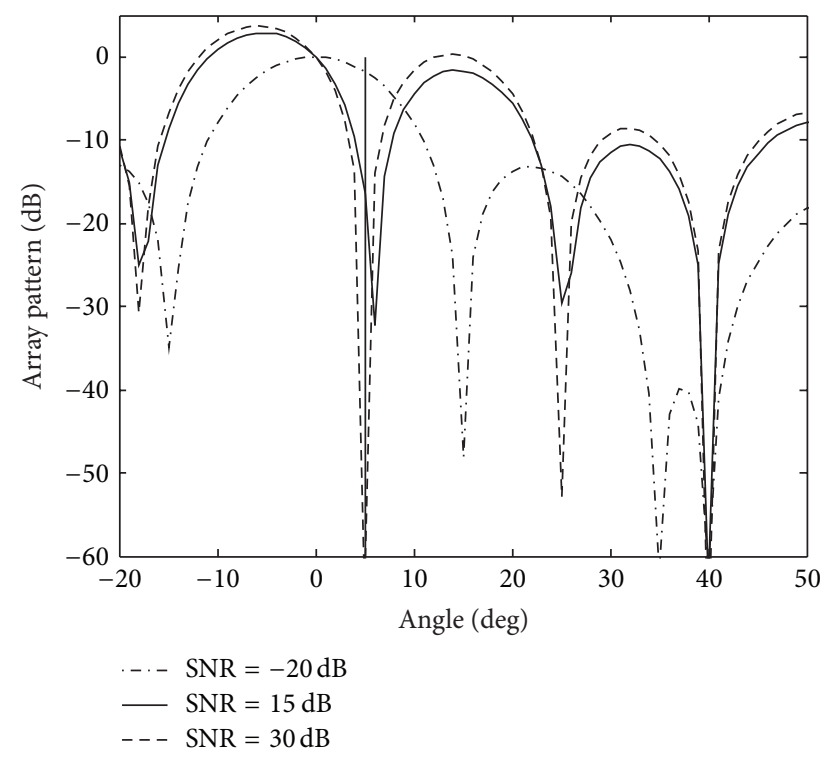

FIGURE 4: Array pattern of Frost beamforming with different SNRs.

so that grating lobes are prevented. The reference DOA of desired signal is $\hat{\theta}_{0}=0^{\circ}$, but the real angle is $\bar{\theta}_{0}=5^{\circ}$. An interference signal incidences from $\theta_{1}=40^{\circ}$ with INR $=$ $20 \mathrm{~dB}$.

We investigate the "self-nulling" phenomenon firstly. When Frost algorithm is used in beamformer, the array patterns are illustrated in Figure 4, where SNR is $-20 \mathrm{~dB}$, $15 \mathrm{~dB}$, and $30 \mathrm{~dB}$, respectively. It shows that the "self-nulling" happens only in the cases of SNR $=15 \mathrm{~dB}$ and $30 \mathrm{~dB}$. That means the weak desired signal will not generate "self-nulling." On the other hand, the null at $\bar{\theta}_{0}$ is deeper if the signal is stronger. But the interference rejection is not impacted seriously for any case.

As mentioned in the previous section, some robust beamforming algorithms can overcome "self-nulling," such as diagonal load [10], eigen structure [12], and doubly constrained Capon beamforming [14]. The next simulation compares them with proposed method through array pattern and computational complexity. Assuming the power of the desired signal is $20 \mathrm{~dB}$ and the other scenarios are unchanged, Figure 5 shows that all these strategies keep the desired signal in the main lobe without "self-nulling." But the new algorithm cannot null the interference, since the phased array model is taken. This effect is also shown by output SINR of array in Table 1. Although the $19.09 \mathrm{~dB}$ is not the best one, the computational complexity of proposed algorithm is much less than other algorithms according to Table 1, where the computing times are obtained by computer with $2.4 \mathrm{GHz}$ CPU and 5000 snapshots. From the engineering point of view, the new method is much more acceptable than the other ones.

Array gain is an important parameter to describe the performance for beamformer, which is defined as

$$
G_{A}=\frac{\text { SINR }_{\text {out }}}{\text { SINR }_{\text {in }}}
$$

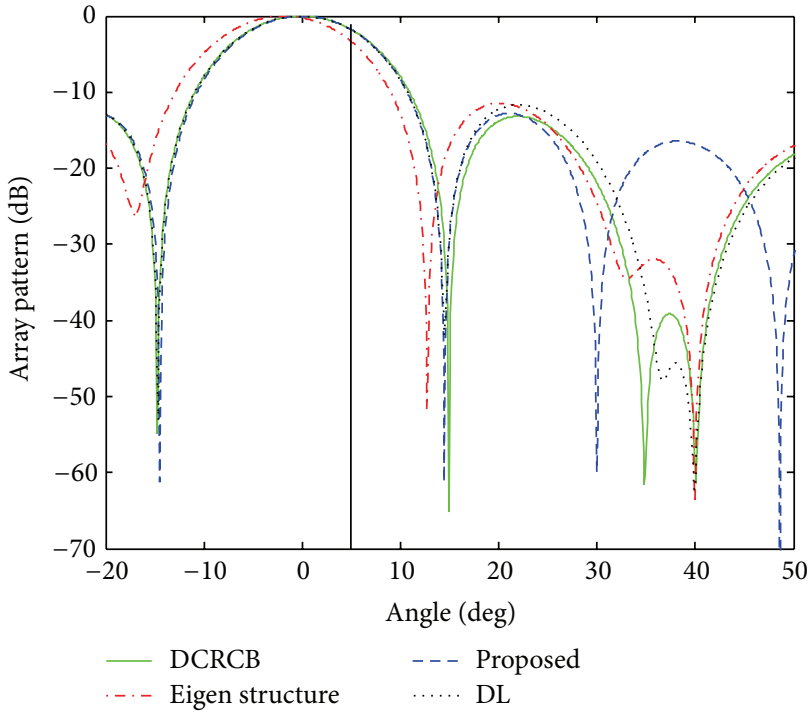

FIGURE 5: Array pattern comparison between different algorithms.

TABLE 1: Output SINR and computational complexity compare between various robust beamformings.

\begin{tabular}{lcc}
\hline Beamforming & Output SINR $(\mathrm{dB})$ & Computing time $(\mathrm{s})$ \\
\hline DCRCB [14] & 24.94 & 0.385771 \\
Eigen structure [12] & 25.61 & 0.014192 \\
Proposed algorithm & 19.09 & 0.009237 \\
Diagonal load [10] & 18.59 & 0.032774 \\
\hline
\end{tabular}

where the SINR $_{\text {out }}$ refers to the SINR of array response $y(t)$ and SINR $_{\text {in }}$ represents the SINR of received signal $x_{m}(t)$ at each sensor. Considering the dynamic range of desired signal from $-20 \mathrm{~dB}$ to $30 \mathrm{~dB}$ and keeping the other scenarios, Figure 6 compares the array gains among the Frost algorithm, idealistic beamformer, phased array, and proposed method. The array gain curve of proposed beamformer consists of two segments locating in low SNR and high SNR regions, respectively. The one at low SNR region is the same as the curve of Frost beamformer. The other at high SNR region is the same as the curve of phased array. The connection point of the two segments is determined by the threshold $\varepsilon$. This can be verified by the simulation where $\varepsilon$ is equal to $1,1.5$, and 2, respectively. As shown in Figure 6, the connection points locate at (BC), $\mathrm{D}$, and (EF), respectively. And $\varepsilon=1.5$ is the optimal threshold with the above assumptions.

\section{Conclusion}

When desired signal is strong, the proposed beamformer switches to phased array model from Frost adaptive array model. It is much easier to be implemented by FPGA or DSPs. But how to choose the threshold $\varepsilon$ needs to be studied further. 


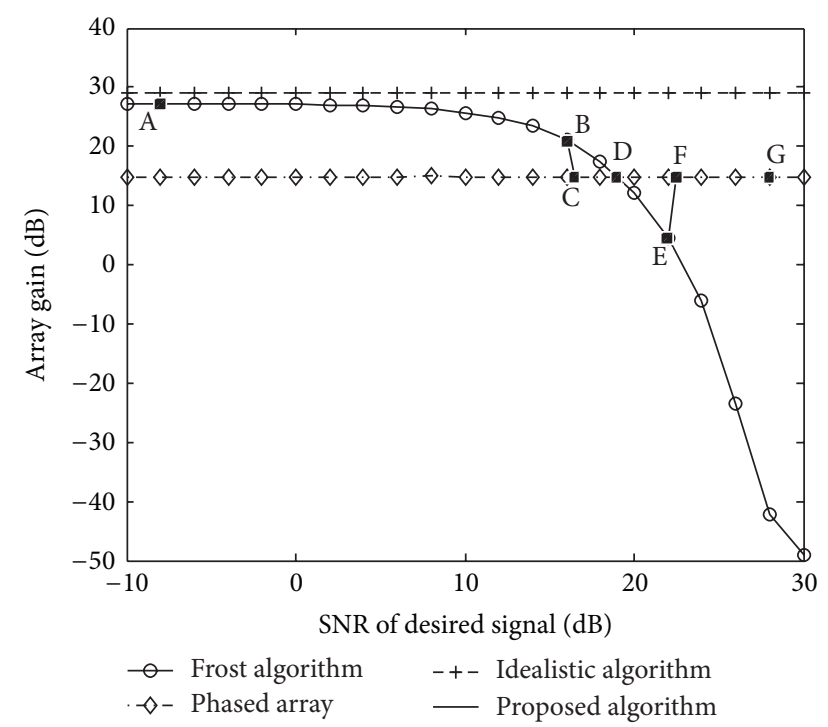

Figure 6: Array gain of proposed algorithm with different thresholds.

\section{Conflict of Interests}

The authors declare that they have no financial and personal relationships with other people or organizations that can inappropriately influence their work; there are no professional or other personal interests of any nature or kind in any product, service, and company that could be construed as influencing the position presented in the paper entitled.

\section{Acknowledgments}

The authors would like to acknowledge the support of the Science and Technology Commission of Chongqing through the Nature Science Fund (2013jjB40005). This work is also supported by the Fundamental Research Funds for the Central University (CDJZR12165501) of China.

\section{References}

[1] O. L. Frost III, "An algorithm for linearly constrained adaptive array processing," Proceedings of the IEEE, vol. 60, no. 8, pp. 926-935, 1972.

[2] M. Wax and Y. Anu, "Performance analysis of the minimum variance beamformer in the presence of steering vector errors," IEEE Transactions on Signal Processing, vol. 44, no. 4, pp. 938947, 1996.

[3] C.-Y. Chen and P. P. Vaidyanathan, "Quadratically constrained beamforming robust against direction-of-arrival mismatch," IEEE Transactions on Signal Processing, vol. 55, no. 8, pp. 41394150, 2007.

[4] H.-S. Lui, H. T. Hui, and M. S. Leong, "A note on the mutualcoupling problems in transmitting and receiving antenna arrays," IEEE Antennas and Propagation Magazine, vol. 51, no. 5, pp. 171-176, 2009.

[5] A. J. Kerkhoff and H. Ling, "A simplified method for reducing mutual coupling effects in low frequency radio telescope phased arrays," IEEE Transactions on Antennas and Propagation, vol. 59, no. 6, pp. 1838-1845, 2011.

[6] W. P. M. N. Keizer, "Fast and accurate array calibration using a synthetic array approach," IEEE Transactions on Antennas and Propagation, vol. 59, no. 11, pp. 4115-4122, 2011.

[7] Y. Li and M. H. Er, “Theoretical analyses of gain and phase error calibration with optimal implementation for linear equispaced array," IEEE Transactions on Signal Processing, vol. 54, no. 2, pp. 712-723, 2006.

[8] A. Young, M. V. Ivashina, R. Maaskant, O. A. Iupikov, and D. B. Davidson, "Improving the calibration efficiency of an array fed reflector antenna through constrained beamforming," IEEE Transactions on Antennas and Propagation, vol. 61, no. 7, pp. 3538-3545, 2013.

[9] H. Pawlak and A. F. Jacob, "An external calibration scheme for DBF antenna arrays," IEEE Transactions on Antennas and Propagation, vol. 58, no. 1, pp. 59-67, 2010.

[10] A. Elnashar, S. M. Elnoubi, and H. A. El-Mikati, "Further study on robust adaptive beamforming with optimum diagonal loading," IEEE Transactions on Antennas and Propagation, vol. 54, no. 12, pp. 3647-3658, 2006.

[11] X. Mestre and M. Á. Lagunas, "Finite sample size effect on minimum variance beamformers: optimum diagonal loading factor for large arrays," IEEE Transactions on Signal Processing, vol. 54, no. 1, pp. 69-82, 2006.

[12] W. S. Youn and C. K. Un, "Robust adaptive beamforming based on the eigenstructure method," IEEE Transactions on Signal Processing, vol. 42, no. 6, pp. 1543-1547, 1994.

[13] S. D. Somasundaram, "Linearly constrained robust Capon beamforming," IEEE Transactions on Signal Processing, vol. 60, no. 11, pp. 5845-5856, 2012.

[14] J. Li, P. Stoica, and Z. Wang, "Doubly constrained robust Capon beamformer," IEEE Transactions on Signal Processing, vol. 52, no. 9, pp. 2407-2423, 2004.

[15] P. Flikkema, "Spread-spectrum techniques for wireless communication," IEEE Signal Processing Magazine, vol. 14, no. 3, pp. 2636, 1997.

[16] H. L. van Trees, Optimum Array Processing-Part IV of Detection, Estimation, and Modulation Theory, Wiley-Interscience, Hoboken, NJ, USA, 2002. 

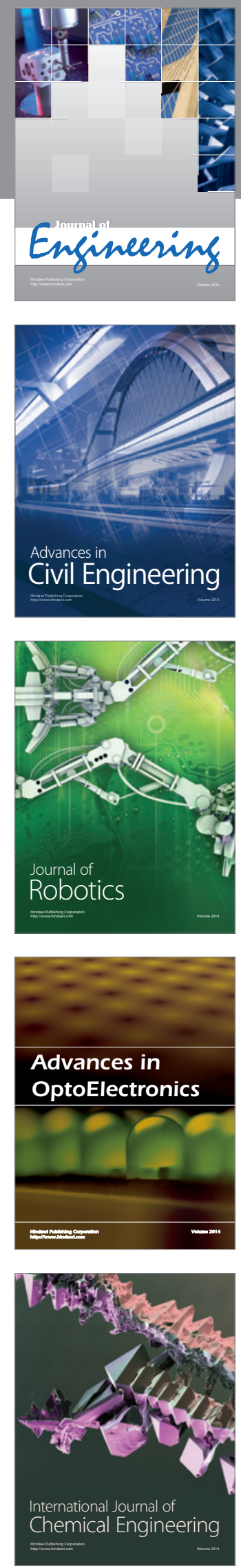

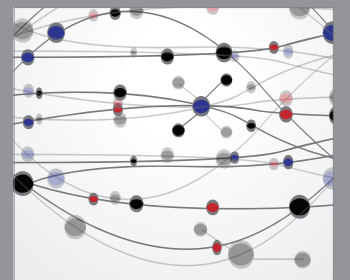

The Scientific World Journal
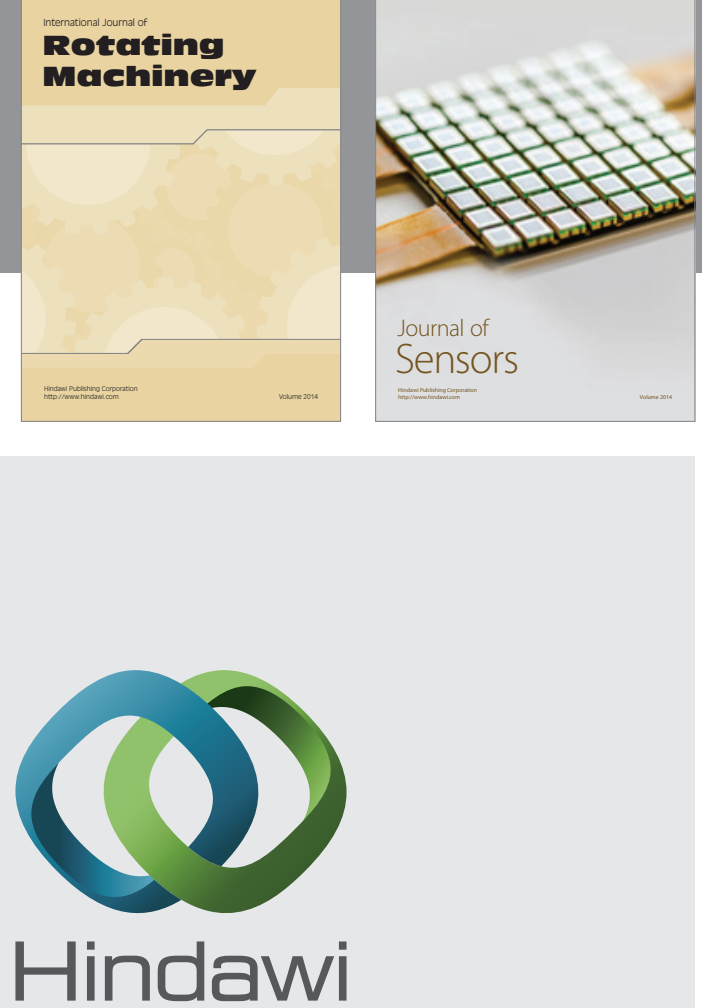

Submit your manuscripts at http://www.hindawi.com
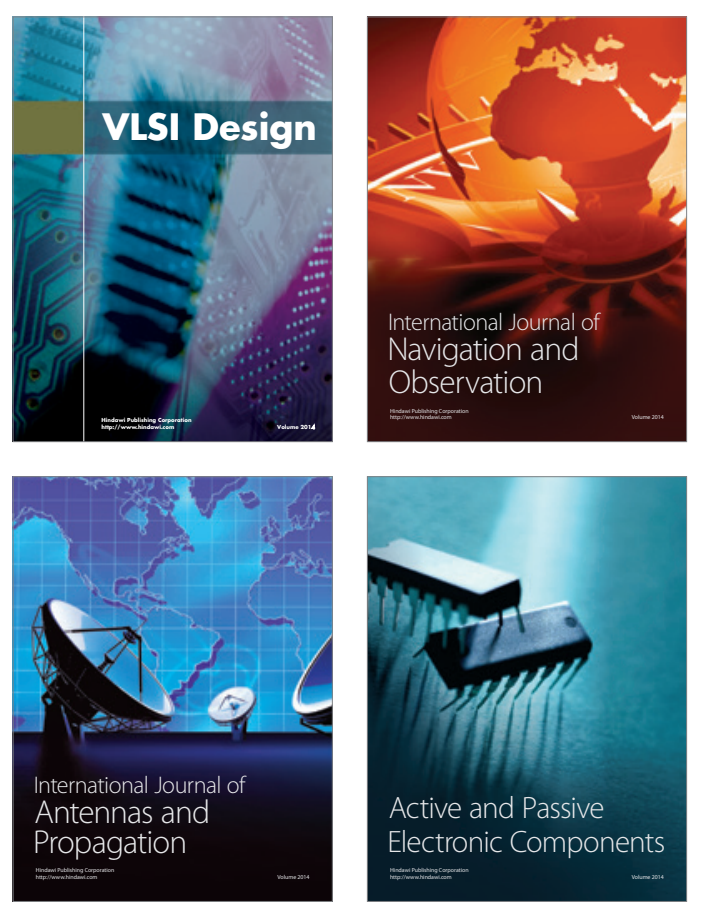
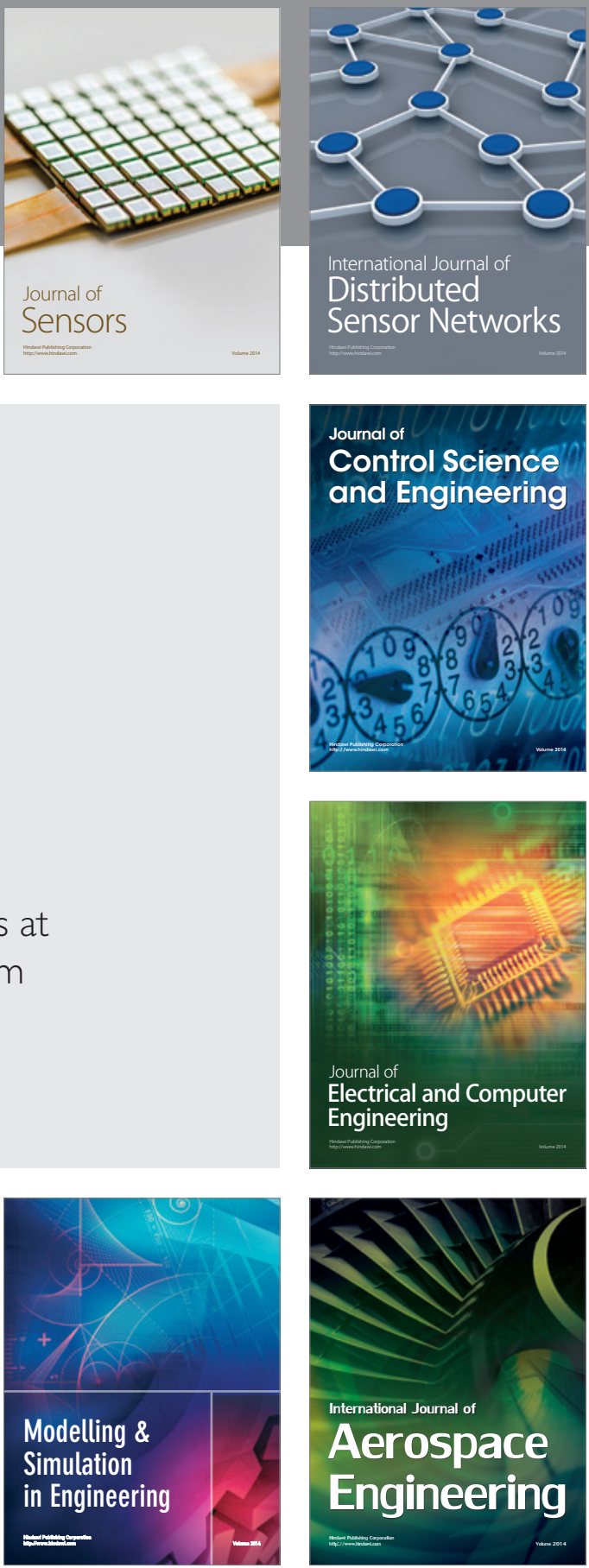

Journal of

Control Science

and Engineering
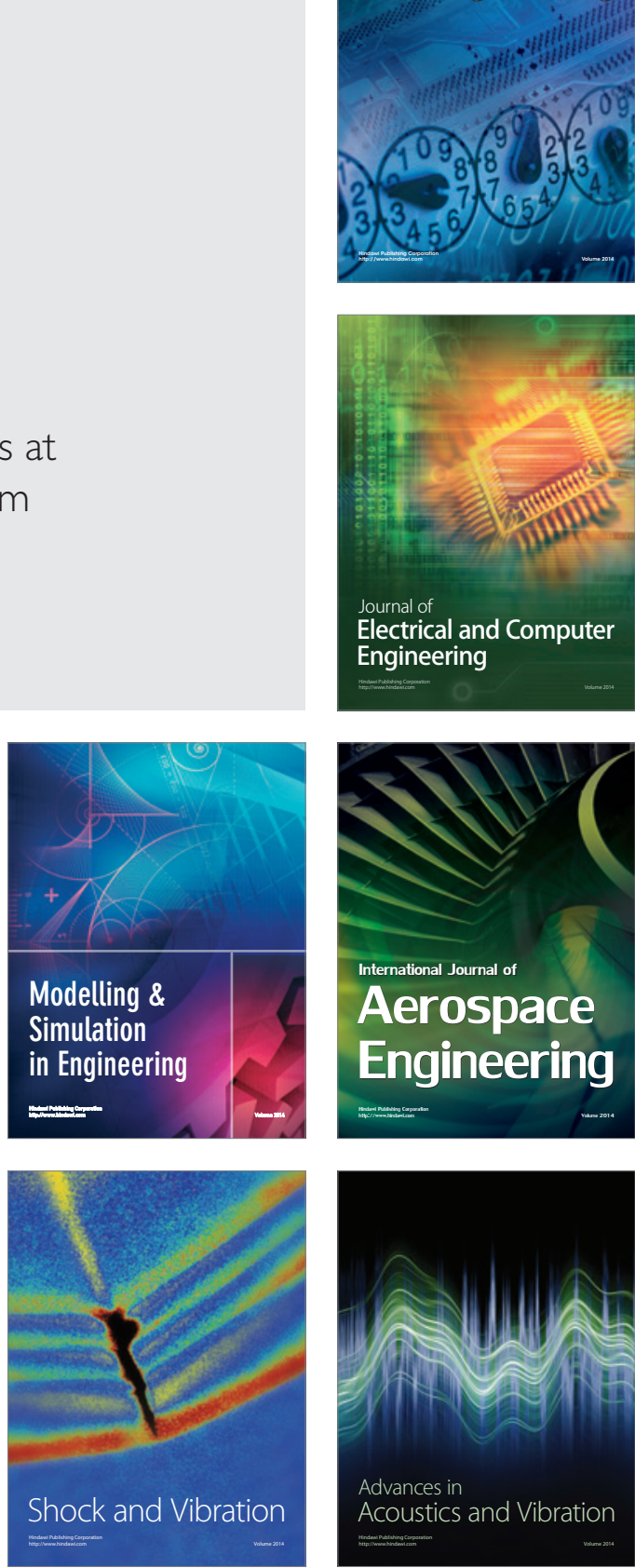